\title{
Inhalt
}

I. Verzeichnis der ausgewerteten Periodika 649

II. Allgemeines 657

Allgemeines - Grundlagen 657. Organisation, Institute, Gesellschaften, Kongresse 659. Bibliographien 661. Handschriftenverzeichnisse, Bestandsverzeichnisse 661. Sammelwerke, Festschriften 663. Wissenschafts- und Gelehrtengeschichte 664

III. Germanische Altertumskunde

IV. Allgemeine und indogermanische Sprachwissenschaft

Sprachphilosophie 670. Allgemeine Sprachwissenschaft 671. Kontrastive Sprachwissenschaft 671. Indogermanische Sprachwissenschaft 677

V. Germanische Sprachen

VI. Ostgermanische Sprachen

VII. Nordgermanische Sprachen

VIII. Westgermanische Sprachen

678

IX. Altniederländisch und Mittelniederländisch 680

X. Deutsch in seiner Gesamtentwicklung 680

XI. Althochdeutsch (8. Jh.-1050) und Altniederdeutsch (9.-12. Jh.) 681

XII. Mittelhochdeutsch (1050-1350) und Mittelniederdeutsch

(13. Jh.-1650)

XIII. Frühneuhochdeutsch (1350-1650)

XIV. Neuhochdeutsch (1650-1945)

XV. Deutsche Sprache nach 1945 bis zur Gegenwart

Allgemeines 689. Phonetik - Phonologie - Graphemik - Orthographie 689. Morphologie - Syntax 690. Semantik - Semiotik 693. Wortbildung - Phraseologie 694. Pragmalinguistik - Textlinguistik - Gesprächsforschung 695. Soziolinguistik - Sprachkritik - Sprachpolitik 700. Psycholinguistik - Spracherwerbsforschung - Sprachverstehensforschung 704. Sprechwissenschaft - Rhetorik - Stilistik 705. Didaktik: Sprache 706

$\begin{array}{ll}\text { XVI. Deutsch als Fremdsprache } & 718\end{array}$

$\begin{array}{ll}\text { XVII. Varietätenlinguistik } & 726\end{array}$

Allgemeines 726. Hochdeutsche und niederdeutsche Mundarten 727. Jiddisch 729. Wissenschafts-, Fach- und Sondersprachen 729

XVIII. Wort- und Namenforschung

Lexikologie 731. Lexikographie 731. Namenforschung 733

XIX. Allgemeines zur Literaturwissenschaft

Allgemeines - Bibliographien - Handbücher 735. Systematik und Methodik 737. Edition und Textkritik 737. Ästhetik - Wertung - Literaturkritik 739. Poetik: Allgemeines, Literaturtheorie 740. Poetik: Gattungen und Arten 746. Poetik: Stilistik und Rhetorik 753. Poetik: Rhetorische Bilder und Figuren 754. Poetik: Stoffe und Motive 755. Poetik: Verslehre - Metrik - Rhythmus 775. Literatur und Gesellschaft 775. Didaktik: Literatur 776

XX. Vergleichende Literaturwissenschaft

Allgemeines 785. Deutsche Literatur und antike Literaturen 786. Deutsche Literatur und romanische Literaturen 786 . Deutsche Literatur und an- 
gelsächsische Literaturen 789. Deutsche Literatur und skandinavische sowie niederländische Literaturen 791. Deutsche Literatur und slawische Literaturen 792. Deutsche Literatur und weitere Literaturen 794

XXI. Nordische Altertumskunde

XXII. Altnordische Literatur

XXIII. Deutsche Literaturgeschichte, Allgemeines

XXIV. Frühmittelalter (770-1170)

Allgemeines 803. Priester Arnolt 804. Ava 804. Christus und die Samariterin 804. Ekkehard IV. von St. Gallen 804. Frutolf von Michelsberg 805 . St. Galler Schularbeit 805 . Heliand 805 . Hildebrandslied 806. Hrotsvit von Gandersheim 806. Kaiserchronik 806. Pfaffe Konrad 807. Pfaffe Lambrecht 807. Malbergische Glossen 807. Notker I. von St. Gallen 808. Notker III. von St. Gallen 808. Otfrid von Weißenburg 808. Ratpert von St. Gallen 808. Rupert von Deutz 808

XXV. Hochmittelalter (1170-1250)

Allgemeines 808. Albrecht von Halberstadt 810. Albrecht von Johannsdorf 810. Eike von Repgow 810. Gottfried von Straßburg 810. Hartmann von Aue 810. Heinrich, Verfasser des Reinhart Fuchs، 811. Heinrich VI., römisch-deutscher Kaiser 811. Heinrich von Morungen 811. Heinrich von dem Türlin 811. Heinrich von Veldeke 811. Herzog Ernst 812. Kudrun 812. Lancelot 812. Moriz von Craûn 812. Neidhart 812. Nibelungenlied 812. Otto von Botenlauben 813. Reinmar der Alte 813. Reinmar von Zweter 813. Der Stricker 813. Der Tannhäuser 813. Tundalus 813. Walther von der Vogelweide 813. Wirnt von Grafenberg 813. Wolfram von Eschenbach 813

XXVI. Spätmittelalter (1250-1450)

Allgemeines 814. Albrecht, Dichter des Jüngeren Titurel 815. Albrecht van Borgunnien 815. Meister Alexander 815. Der arme Konrad 815. Dalimil, deutsch 815. Dietrich von Bocksdorf 816. Dietrich von der Glesse 816 . Dietrichs Flucht 816 . Dorothea 816. Düdesche Arstedie 816. Ebendorfer, Thomas 816. Ebner, Christine 816. Ebner, Margareta 816. Meister Eckhart 816. Elhen von Wolfhagen, Tilemann 816. Elisabeth von Nassau-Saarbrücken 816. Ferguut 817. Der Frankfurter 817. Frauenlob 817. Hartlieb, Johannes 818. Hein van Aken 818. Heinrich von München 818. Hugo von Trimberg 818. Jans Enikel 818. Johann von Würzburg II 819. Johannes von Brakel 819. Karlmeinet 819. Der von Kolmas 819. Konrad von Megenberg 820. Konrad von Würzburg 820. Kremsmünsterer 〈schlesisches> Dorotheenspiel 820. Laurin 820. Leipziger Drogenkompendium 821. Livländische Reimchronik 821. Maastrichter <ripuarisches> Passionsspiel 821. Magdeburger Fragen (und Magdeburger Urteile) 821. Magdeburger Rechtsbücher 821. Mechthild von Magdeburg 821. Meißner Rechtsbuch 821. Michael de Leone 821. Die Minneburg 822. Neidhartspiele 822. Nikolaus von Kues 822. Ortolf von Baierland 822. Oswald von Wolkenstein 822. Reinfried von Braunschweig 822. Der Rosendorn 822. Rosenplüt, Hans 822. Ruprecht von Würzburg 822. Seuse, Heinrich 822. Stromer, Ulman 823. Süßkind von Trimberg 823. Ulrich von Liechtenstein 823. Väterbuch 823. Valentin und Namelos 823. Vierundzwanzig-Paragraphen-Text 823. Wernher der Gartenaere 823. Wienhäuser Osterspiel 823. Wittenwiler, Heinrich 824 . Vom Würzburger Städtekrieg 824

XXVII. Humanismus (1450-1520)

Allgemeines 824. Abdinghofer Arzneibuch 824. Alsfelder Passionsspiel 824. Ambraser Heldenbuch 825. Augsburger Georgsspiel 825. Aventinus, Johannes 825. Der Boiffen Orden 825. Bruno, Chris- 
toph 825. Celtis, Konrad 825. Dietrich von Plieningen 825. Donaueschinger Passionsspiel 825. Dürer, Albrecht 825. Egerer Passionsspiel 825. Emser, Hieronymus 825. Fabri, Felix 825. Folz, Hans 826. Fortunatus 826. Füetrer, Ulrich 826. Gart der Gesundheit 826. Geiler von Kaysersberg, Johannes 826. Gersdorff, Hans von 826. Heinrich von Kettenbach 826. Hutten, Ulrich von 826. Immessen, Arnold 826. Jude von Salms 827. Murner, Thomas 827. Osnabrücker Osterspiel 827. Piccolomini, Aeneas Silvius 827. Preining, Jörg 827. Püterich von Reichertshausen, Jakob 827. Redentiner Osterspiel 827. Rülein von Calw, Ulrich 827. Spengler, Lazarus 828. Tristrant und Isalde 828. Virdung, Sebastian 828 . Wilhelm von Österreich 828

\section{Reformationszeit (1520-1580)}

Allgemeines 828. Balticus, Martinus 830. Berlichingen, Götz von 830. Dobereiner, Philipp 830. Eberlin von Günzburg, Johann 830. Eck, Johannes 831. Fischart, Johann 831. Flacius Illyricus, Matthias 831. Franck, Sebastian 832. Helth, Caspar 832. Herberstein, Sigismund von 832. Herold, Johannes 832. Hunger, Wolfgang 832. Ickelsamer, Valentin 832. Karlstadt, Andreas 833. Königsberger Fastnachtspiel 833. Lemnius, Simon 833. Losch, Jonas 833. Luther, Martin 833. Melanchthon, Philipp 835. Paracelsus 837. Sachs, Hans 837. Schaidenreisser, Simon 837. Staden, Hans 837. Sturmer, Balthasar 837. Thurneisser zum Thurn, Leonhard 837. Wier, Johannes 837. Wild, Sebastian 837. Ziegler, Hieronymus 838 . Zwingli, Huldrych 838

\section{Barock (1580-1700)}

Allgemeines 838. Abraham a Sancta Clara 841. Albertinus, Aegidius 841. Andreae, Johann Valentin 841. Angelus Silesius 841. Balde, Jacob 841. Bidermann, Jakob 841. Birken, Sigmund von 841. Bissel, Johannes 841. Brummer, Johannes 841. Christian II. von Anhalt-Bernburg 841. Drexel, Jeremias 842. Elisabeth Charlotte von Orléans 842. Franckenberg, Abraham von 842. Gallanda, Johann Baptist 842. Geminianus Monacensis 842. Gerhard, Johann 842. Greiffenberg, Catharina Regina von 842. Grimmelshausen, Hans Jakob Christoffel von 842. Gryphius, Andreas 843. Happel, Eberhart Werner 843. Harsdörffer, Georg Philipp 843. Khuen, Johannes 843. Kuhlmann, Quirinus 844. Lalebuch 844. Lohenstein, Daniel Casper von 844. Martin von Cochem 844. Neukirch, Benjamin 844. Oertel, Hieronymus 844. Opitz, Martin 844. Placcius, Vincent 844. Rader, Matthäus 844. Rauscher, Wolfgang 844. Reuter, Christian 844. Richter, Christian 845. Rinckart, Martin 845. Rist, Johann 845. Schweigger, Salomon 846. Speer, Daniel 846. Stubenberg, Johann Wilhelm von 846. Sudermann, Daniel 846. Thamm, Balthasar 846. Tympius, Matthaeus 847. Waldung, Wolfgang 847. Weise, Christian 848. Witekind, Hermann 848. Zahn, Zacharias 848

\section{Aufklärung, Empfindsamkeit (1700-1770)}

Allgemeines 849. Bohse, August 851. Breitenbauch, Georg August von 851. Brockes, Barthold Heinrich 851. Gerstenberg, Heinrich Wilhelm von 851. Geßner, Salomon 851. Gottsched, Johann Christoph 851. Gressel, Johann Georg 851. Haller, Albrecht von 851. Hamann, Johann Georg 851. Hunold, Christian Friedrich 852. Klopstock, Friedrich Gottlieb 852. König, Johann Ulrich von 852. Krüger, Johann Gottlob 852. Lessing, Gotthold Ephraim 852. Mendelssohn, Moses 853. Möser, Justus 853. Neumayr, Franz 853. Rost, Johann Christoph 853. Schlegel, Johann Elias 853. Schnabel, Johann Gottfried 853. Thomasius, Christian 853. Trenck, Friedrich von der 854. 
Uz, Johann Peter 854. Weiße, Christian Felix 854. Winckelmann, Johann Joachim 854. Wolff, Christian 854. Zachariae, Justus Friedrich Wilhelm 854

XXXI. Sturm und Drang, Klassik, Romantik (1770-1830)

Allgemeines 854. Arndt, Ernst Moritz 859. Arnim, Achim von 859. Arnim, Bettina von 859. Assing, David 859. Assing, Rosa Maria 859. Baggesen, Jens 860. Bahrdt, Carl Friedrich 860. Bernhardi, August Ferdinand 860 . Bertuch, Friedrich Johann Justin 860 . Blanckenburg, Christian Friedrich von 860. Bouterwek, Friedrich 860. Brenner, Anton Jakob 860. Brentano, Clemens 860. Bucher, Anton von 861. Campe, Joachim Heinrich 861. Carolath-Beuthen, Adelheid von 861. Chamisso, Adelbert von 861. Eichendorff, Joseph von 861. Engel, Johann Jakob 862. Eschenburg, Johann Joachim 862. Falk, Johann Daniel 862. Fallmerayer, Jakob Philipp 862. Fischer, Caroline Auguste 862. Follen, Karl 862. Forster, Georg 862. Fouqué, Friedrich de la Motte 862. Franul von Weißenthurn, Johanna 863. Gallitzin, Adelheid Amalia von 863. Garve, Christian 863. Goethe, Johann Wolfgang von 863. Gräffer, Franz 869. Grimm, Jacob 869. Grimm, Wilhelm 869. Günderrode, Karoline von 869. Halem, Gerhard Anton von 869. Hayn, Juliane 870. Hebel, Johann Peter 870. Hegel, Georg Wilhelm Friedrich 870. Heinse, Wilhelm 870. Herder, Johann Gottfried 870. Hölderlin, Friedrich 873. Hoffmann, Ernst Theodor Amadeus 875. Huber, Therese 877. Humboldt, Alexander von 877 . Humboldt, Wilhelm von 877. Iffland, August Wilhelm 878. Jean Paul 878. Jelačić von Bužim, Joseph 878. Kleist, Heinrich von 878. Knigge, Adolph von 881. Kobbe, Theodor von 883. Körner, Christian Gottfried 883. Körner, Theodor 883. Komareck, Johann Nepomuk 883. Kotzebue, August von 883. Kratter, Franz 883. La Roche, Sophie von 883. Lavater, Johann Caspar 884. Lenz, Jakob Michael Reinhold 884. Lichtenberg, Georg Christoph 884. Mailáth, Johann 884. Mauvillon, Jakob 884. Meißner, August Gottlieb 885. Merck, Johann Heinrich 885. Merkel, Garlieb Helwig 885. Moritz, Karl Philipp 885. Mozart, Wolfgang Amadeus 885. Müller, Johann Gottwerth 885. Müller, Wilhelm 〈Griechen-Müller〉 885. Naubert, Benedikte 886. Nicolai, Friedrich 886. Nonne, Johann Gottfried Christian 886. Novalis 886. Pezzl, Johann 886. Platen, August von 886. Pustkuchen, Johann Friedrich Wilhelm 887. Pyrker von Oberwart, Johann Ladislaus 887. Rambach, Friedrich Eberhard 887. Rebmann, Andreas Georg Friedrich 887. Ritter, Johann Wilhelm 887. Schelling, Friedrich Wilhelm Joseph von 887. Schenkendorf, Max von 887. Schiller, Charlotte von 887. Schiller, Friedrich von 887. Schlegel, August Wilhelm 890. Schlegel, Christiane Karoline 890. Schlegel, Friedrich 890. Schleiermacher, Friedrich Daniel Ernst 891. Schneider, Karl Agnel 891. Schubart, Christian Friedrich Daniel 891. Schubert, Gotthilf Heinrich 891. Schütz, Friedrich Wilhelm von 891. Schulze, Ernst 891. Seume, Johann Gottfried 891. Steffens, Henrik 892. Stein, Charlotte von 892. Temme, Jodocus Donatus Hubertus 892. Tieck, Ludwig 892. Ueltzen, Hermann Wilhelm Franz 893. Unger, Friederike Helene 893. Unzer, Ludwig August 893. Varnhagen von Ense, Karl August 893. Varnhagen von Ense, Rahel 894. Vogt, Nicolaus 894. Voß, Johann Heinrich 894. Voß, Julius von 894. Wackenroder, Wilhelm Heinrich 894. Weidig, Friedrich Ludwig 894. Werner, Zacharias 894. Wessenberg, Ignaz Heinrich von 894. Westenrieder, Lorenz von 894. Wetzel, Friedrich Gottlob 894. Wezel, Johann Karl 895. Wieland, Christoph Martin 895. Wolzogen, Caroline von 896. Zschokke, Heinrich 896 
Allgemeines 896. Alexis, Willibald 899. Anneke, Mathilde Franziska 899. Anzengruber, Ludwig 899. Assing, Ludmilla 899. Auerbach, Berthold 899. Bernstein, Karl Hugo Amber 899. Börne, Ludwig 899. Büchner, Georg 899. Burckhardt, Jacob 900. Busch, Wilhelm 900. Dronke, Ernst 900. Droste-Hülshoff, Annette von 900. Düringsfeld, Ida von 901. Ebersberg, Ottokar Franz 901. Ebert, Karl Egon 901. Ellissen, Adolf 901. Engels, Friedrich 901. Eschwege, Wilhelm von 901. Felder, Franz Michael 901. Fontane, Theodor 901. Freiligrath, Ferdinand 905. Freytag, Gustav 906. Gerstäcker, Friedrich 907. Glaßbrenner, Adolf 907. Goedsche, Hermann Ottomar Friedrich 907. Goethe, Ottilie von 907. Grabbe, Christian Dietrich 907. Grillparzer, Franz 907. Groth, Klaus 908. Gutzkow, Karl 909. Hackländer, Friedrich Wilhelm 910. Haffner, Karl 910. Hahn-Hahn, Ida von 910. Hasert, Friedrich Reinhold 910. Hebbel, Friedrich 910. Heine, Heinrich 911. Heyse, Paul 911. Hillern, Wilhelmine von 913. Hoffmann von Fallersleben, August Heinrich 913. Horn, W. O. von 914. Immermann, Karl Leberecht 914. Keller, Gottfried 914. Kerner, Justinus 914. Kompert, Leopold 914. Kurz, Hermann 915. Laube, Heinrich 915. Lewald, Fanny 915. Marx, Karl 916. Menzel, Wolfgang 916. Merckel, Wilhelm von 916. Meyer, Conrad Ferdinand 917. Mörike, Eduard 917. Mügge, Theodor 918. Mühlbach, Luise 918. Mundt, Theodor 918. Nestroy, Johann 918. Niemann, August 918. Nietzsche, Friedrich 918. Ortlepp, Ernst 920. Otto-Peters, Louise 921. Penn, Heinrich 921. Pfeiffer, Ida 921. Pfundheller, Josef 921. Pietsch, Ludwig 921. Pocci, Franz von 921. Preradović, Petar 921. Prutz, Robert 921. Pückler-Muskau, Hermann von 921. Raabe, Wilhelm 921. Ring, Max 922. Rückert, Friedrich 922. Scherenberg, Christian Friedrich 923. Schiff, Theodor 923. Schirmer, Adolf 923. Schloenbach, Carl Arnold 923. Schmidt, Julian 923. Schnezler, August 923. Schopenhauer, Arthur 923. Schwarzenberg, Friedrich zu 923. Sealsfield, Charles 923. Spielhagen, Friedrich 925. Stahr, Adolf 925. Stelzhamer, Franz 925. Steub, Ludwig 925. Stifter, Adalbert 925. Storm, Theodor 927. Strauß, David Friedrich 929. Uhl, Friedrich 932. Vischer, Friedrich Theodor 932. Wagner, Richard 932. Weber, Vinzenz P. 932. Weerth, Georg 932. Wenzig, Josef 932. Wienbarg, Ludolf 932. Willkomm, Ernst Adolf 933. Zeise, Karl Heinrich Theodor 933

\section{Vom Naturalismus bis 1945}

Allgemeines 933. Einzelne Abschnitte 935. Lyrik 942. Epik 942. Drama 942. Achleitner, Arthur 942. Altenberg, Peter 943. Arlt, Wilhelm von 943. Asch, Schalom 943. Bahr, Hermann 943. Ball, Hugo 943. Basil, Otto 944. Baudissin, Eva von 944. Baum, Vicki 944. Becher, Johannes R. 944. Beer, Natalie 944. Benjamin, Walter 944. Benn, Gottfried 946. Benrath, Henry 947. Bernstein, Elsa 947. Bierbaum, Otto Julius 947. Binding, Rudolf G. 947. Birnbaum, Nathan 947. Blei, Franz 947. Bloch, Ernst 948. Böhlau, Helene 948. Bonsels, Waldemar 948. Borchardt, Rudolf 948. Brachvogel, Carry 948. Brahm, Otto 948. Brecht, Bertolt 949. Brehm, Bruno 953. Breuer, Isaac 953. Britting, Georg 953. Broch, Hermann 953. Brod, Max 955. Bronnen, Arnolt 955. Bruck-Auffenberg, Natalie 955. Buber, Martin 955. Buschbeck, Erhard 955. Carossa, Hans 955. Chiavacci, Vinzenz 956. Christ, Lena 956. Däubler, Theodor 956. Dauthendey, Max 956. Dehmel, Richard 956. Delle Grazie, Marie Eugenie 956. Doderer, Heimito von 956. Döblin, Alfred 959. Dohm, Hedwig 959. Druskowitz, Helene von 959. Dwinger, Edwin Erich 959. Ebner-Eschenbach, Marie 
von 959. Eckstein, Ernst 959. Egidy, Emmy von 959. Ehrenstein, Albert 959. Einstein, Carl 959. Eisler, Hanns 960. Eisner, Kurt 960. Engelke, Gerrit 962. Ernst, Max 962. Eucken, Rudolf 962. Ewers, Hanns Heinz 962. Feuchtwanger, Lion 962. Freud, Sigmund 962. Friedell, Egon 963. Gagarin, Eugen 963. Ganghofer, Ludwig 963. George, Stefan 963. Gilbert, Robert 964. Glauser, Friedrich 964. Gleit, Maria 964. Gneisenau, Maria von 965. Goldberg, Lea 965. Goll, Yvan 965. Graf, Oskar Maria 965. Grimm, Hans 965. Grisar, Erich 965. Gronemann, Sammy 965. Groner, Auguste 965. Gurk, Paul 965. Haas, Willy 965. Haecker, Theodor 965. Hartlaub, Felix 966. Hartleben, Otto Erich 966. Hartwig, Mela 966. Hauptmann, Gerhart 966. Hausdorff, Felix 967. Haushofer, Marie 967. Haushofer, Max 967. Haushofer-Merk, Emma 967. Hausmann, Raoul 967. Heidegger, Martin 968. Heilig, Bruno 968. Heitz, Markus 968. Heller, Leo 968. Herzl, Theodor 968. Hesse, Hermann 969. Hessel, Franz 970. Heym, Georg 970. Hille, Peter 970. Hiller, Kurt 970. Hofmannsthal, Hugo von 970. Holz, Arno 972. Horváth, Ödön von 972. Huch, Ricarda 972. Huelsenbeck, Richard 972. Jahnn, Hans Henny 973. Jaksch, Friedrich 973. Janitschek, Maria 973. Janowitz, Hans 973. Jaspers, Karl 973. Johannsen, Ernst 973. Jünger, Ernst 973. Jünger, Friedrich Georg 974. Kadich von Pferd, Hans 974. Kästner, Erich 974. Kafka, Franz 974. Kahler, Erich von 978. Kahn, Arthur 978. Kaléko, Mascha 978. Kaltneker, Hans 979. Kantorowicz, Gertrud 979. Kayser, Rudolf 979. Kellermann, Bernhard 979. Kessler, Harry 979. Keun, Irmgard 979. Klages, Ludwig 979. Klee, Paul 979. Klemm, Wilhelm 979. Kohlrausch, Robert 980. Kokoschka, Oskar 980. Kolb, Annette 980. Kolbenheyer, Erwin Guido 980. Kommerell, Max 980. Kracauer, Siegfried 981. Kraus, Karl 982. Kubin, Alfred 982. Kuh, Anton 982. Lampe, Friedo 982. Lamszus, Wilhelm 983. Landshoff-Yorck, Ruth 984. Langer, Georg 984. LaskerSchüler, Else 984. Laßwitz, Kurd 984. Lautensack, Heinrich 985. Le Fort, Gertrud von 985. Leitner, Maria 985. Leivick, H. 985. Leppin, Paul 985. Lernet-Holenia, Alexander 985. Lichtenstein, Alfred 985. Liebhard, Franz 985. Lindau, Paul 985. Loerke, Oskar 986. Lucka, Emil 986. Ludwig, Emil 986. Mahler-Werfel, Alma 986. Mann, Erika 986. Mann, Heinrich 986. Mann, Klaus 986. Mann, Thomas 987. May, Karl 992. Mayreder, Rosa 993. Meerbaum-Eisinger, Selma 993. Meschendörfer, Adolf 993. Meyrink, Gustav 993. Michel, Robert 993. Mimra, Robert 994. Mitterer, Erika 994. Möller, Karl von 994. Mühsam, Erich 994. Müller, Anton <Bruder Willram> 995. Müller, Robert 995. Müller-Frerich, Franz 995. Musil, Robert 995. Neumann, Robert 996. Neustädter, Erwin 997. Nolde, Emil 997. Nordau, Max 997. Nowak, Heinrich 997. Oppenheimer, Friedrich 997. Ott, Erwin 997. Pannwitz, Rudolf 997. Perutz, Leo 997. Picard, Max 997. Pichler, Anton 997. Podewils, Clemens 997. Pötzl, Eduard 997. Polte, Paul 997. Preradović, Paula von 998. Pringsheim, Hedwig 998. Queri, Georg 998. Reck-Malleczewen, Friedrich Percyval 998. Remarque, Erich Maria 999. Reuter, Gabriele 999. Reventlow, Franziska zu 999. Richter, Johannes 999. Rilke, Rainer Maria 999. Ringelnatz, Joachim 1001. Robakidse, Grigol 1001. Rode, Walther 1001. Rosegger, Peter 1001. Rosenfeld, Oskar 1001. Rosenkranz, Moses 1001. Rosenzweig, Franz 1001. Roth, Joseph 1002. Rothgießer, Fritz 1004. Ruederer, Josef 1004. Saar, Ferdinand von 1004. Salomon, Ernst von 1004. Salten, Felix 1004. Schaffner, Jakob 1004. Schaper, Edzard 1004. Schlaf, Johannes 1004. Schlick, Heinz 1004. Schmitt, Carl 1004. Schneider, Reinhold 1005. Schnitzler, 
Arthur 1005. Schönberg, Arnold 1006. Schoßleitner, Karl 1006. Schullern, Heinrich von 1006. Schwarzenbach, Annemarie 1006. Seelig, Carl 1006. Seghers, Anna 1006. Seidel, Ina 1007. Serner, Walter 1008. Sieburg, Friedrich 1008. Siegelberg, Mark 1008. Sigerus, Emil 1008. Singer, Oskar 1008. Siodmak, Curt 1008. Soyfer, Jura 1008. Spengler, Oswald 1009. Spyri, Johanna 1009. Sternheim, Carl 1009. Stomps, Victor Otto 1009. Stramm, August 1009. Strauss, Richard 1009. Strobl, Karl Hans 1009. Sudermann, Hermann 1010. Suhrkamp, Peter 1010. Susman, Margarete 1010. Suttner, Bertha von 1010. Tetzner, Lisa 1010. Thieß, Frank 1010. Thoma, Ludwig 1010. Toller, Ernst 1010. Torberg, Friedrich 1010. Trakl, Georg 1010. Traven, B. 1012. Trenker, Luis 1012. Tucholsky, Kurt 1012. Uiberacker, Ernst Josef 1012. Ullmann, Regina 1012. Urbanitzky, Grete von 1012. Usinger, Fritz 1013. Valentin, Karl 1013. Vegesack, Siegfried von 1013. Vollmoeller, Karl Gustav 1013. Vukelich, Wilma von 1013. Walser, Robert 1013. Wantoch, Susanne 1017. Wedding, Alex 1017. Wedekind, Frank 1017. Weiskopf, Franz Carl 1017. Weiß, Ernst 1017. Werfel, Franz 1018. Wichner, Josef 1018. Wiechert, Ernst 1018. Winder, Ludwig 1018. Winkler, Eugen Gottlob 1018. Winsloe, Christa 1018. Wittgenstein, Ludwig 1019. Wittig, Joseph 1019. Wittstock, Erwin 1019. Wolf, Friedrich 1019. Wrede, Friedrich von 1019. Wulffen, Erich 1019. Zech, Paul 1019. Ziegler, Regine 1020. Zillich, Heinrich 1020. Zuckerkandl, Berta 1020. Zuckmayer, Carl 1020. Zweig, Arnold 1020. Zweig, Stefan 1020

XXXIV. Literatur von 1945 bis 1989

Allgemeines 1021. Einzelne Abschnitte 1025. Lyrik 1026. Epik 1027. Drama 1028. Achternbusch, Herbert 1028. Adler, Hans Günther 1028. Adorno, Theodor W. 1028. Aichinger, Ilse 1029. Aloni, Jenny 1030. Améry, Jean 1030. Anders, Günther 1030. Anders, Richard 1030. Andersch, Alfred 1030. Andres, Stefan 1032. Anger-Schmidt, Gerda 1032. Aparicio, Guillermo 1032. Apitz, Bruno 1032. Arendt, Erich 1032. Arendt, Hannah 1032. Astel, Arnfrid 1032. Atabay, Cyrus 1032. Augustin, Ernst 1033. Ausländer, Rose 1033. Bachmann, Ingeborg 1034. Bauer, Wolfgang 1035. Baukloh, Friedhelm 1035. Bayer, Konrad 1035. Becker, Jürgen 1035. Becker, Jurek 1036. Berger, Herbert 1036. Bernhard, Thomas 1036. Bichsel, Peter 1037. Bienek, Horst 1038. Biermann, Wolf 1038. Birkner, Andreas 1038. Blumenberg, Hans 1038. Bobrowski, Johannes 1038. Böll, Heinrich 1038. Boie, Kirsten 1039. Borchert, Wolfgang 1039. Born, Nicolas 1039. Bracharz, Kurt 1039. Brambach, Rainer 1039. Brandhorst, Andreas 1039. Brasch, Thomas 1039. Braun, Volker 1039. Brežná, Irena 1040. Brinkmann, Rolf Dieter 1040. Brock, Peter 1040. Bröger, Achim 1041. Bulkowski, Hansjürgen 1041. Burger, Hermann 1041. Burmeister, Brigitte 1041. Buselmeier, Michael 1041. Canetti, Elias 1042. Celan, Paul 1042. Chobot, Manfred 1044. Claus, Carlfriedrich 1044. Dean, Martin R. 1044. Degener, Volker W. 1044. Degenhardt, Franz Josef 1045. Dikmen, Şinasi 1045. Dischereit, Esther 1045. Döhl, Reinhard 1045. Dörnemann, Kurt 1045. Domin, Hilde 1045. Dor, Milo 1045. Dorst, Tankred 1045. Drozdowski, Georg 1046. Duden, Anne 1046. Dürrenmatt, Friedrich 1046. Duvanel, Adelheid 1046. Egel, Karl Georg 1046. Eich, Günter 1046. Eichberger, Günter 1046. Elbogen, Paul 1047. Elliger, Katharina 1047. Elsner, Gisela 1047. Ende, Michael 1047. Enzensberger, Hans Magnus 1048. Ernst, Gustav 1048. Fassbinder, Rainer Werner 1049. Federmann, Reinhard 1050. Federspiel, Jürg 1050. Fichte, Hubert 1050. Filip, Ota 1050. Franke, Herbert W. 1050. Fries, Fritz Rudolf 1050. Frisch, Max 1050. Frischmuth, Barbara 1051. Fritz, Marianne 1051. Fuchs, Jürgen 1051. Fühmann, Franz 1054. Fürnberg, Louis 1054. Gabriel, Ul- 
rich 1054. Gahse, Zsuzsanna 1054. Geerk, Frank 1054. Geiser, Christoph 1055. Genazino, Wilhelm 1055. Gernhardt, Robert 1055. Göhre, Frank 1055. Gölzenleuchter, Horst Dieter 1056. Goes, Albrecht 1056. Goetz, Rainald 1056. Goldschmidt, Georges-Arthur 1056. Goldt, Max 1056. Gomringer, Eugen 1056. Gong, Alfred 1056. Grass, Günter 1056. Gross, Walter 1058. Gruber, Reinhard P. 1058. Grün, Max von der 1058. Grüning, Uwe 1058. Gstrein, Norbert 1058. Günther, Egon 1058. Guggenmos, Josef 1059. Haas, Wolf 1059. Hackl, Erich 1059. Hänny, Reto 1059. Härtling, Peter 1059. Hahn, Ulla 1059. Hamm, Peter 1060. Handke, Peter 1060. Haneke, Michael 1061. Harig, Ludwig 1061. Hartinger, Ingram 1061. Hartmann, Lukas 1061. Hartung, Harald 1062. Haushofer, Marlen 1062. Heckmann, Herbert 1062. Heiduczek, Werner 1062. Hein, Christoph 1062. Heinz, Franz 1062. Heißenbüttel, Helmut 1062. Held, Kurt 1062. Helfer, Monika 1063. Helmlé, Eugen 1063. Henisch, Peter 1063. Henscheid, Eckhard 1063. Henze, Hans Werner 1063. Hermlin, Stephan 1063. Heym, Stefan 1063. Hilbig, Wolfgang 1063. Hildesheimer, Wolfgang 1063. Hilsenrath, Edgar 1064. Hochgatterer, Paulus 1064. Hochhuth, Rolf 1064. Höllerer, Walter 1064. Hohl, Ludwig 1064. Hohler, Franz 1064. Honigmann, Barbara 1064. Horbelt, Rainer 1064. Horkheimer, Max 1064. Huchel, Peter 1065. Jägersberg, Otto 1065. Jandl, Ernst 1065. Jansen, Erich 1066. Jelinek, Elfriede 1066. Jeschke, Wolfgang 1067. Johannimloh, Norbert 1067. Johnson, Uwe 1067. Jokostra, Peter 1067. Jonke, Gert 1067. Just, Gustav 1067. Käufer, Hugo Ernst 1067. Kant, Hermann 1068. Kaschnitz, Marie Luise 1068. Kempowski, Walter 1068. Kessemeier, Siegfried 1068. Kirchhoff, Bodo 1068. Kleeberg, Michael 1069. Klein, Ewalda 1069. Kling, Thomas 1069. Kluge, Alexander 1069. Koch, Valeria 1071. Köhlmeier, Michael 1071. Koeppen, Wolfgang 1071. Körner, Wolfgang 1071. Kolbe, Uwe 1072. Kordon, Klaus 1072. Korn, Renke 1072. Kriwet, Ferdinand 1072. Kroetz, Franz Xaver 1072. Kunert, Günter 1072. Kunze, Reiner 1072. Kurzeck, Peter 1072. Lange, Hartmut 1073. Langgässer, Elisabeth 1073. Langhammer, Leopold 1073. Lebert, Hans 1073. Ledig, Gert 1073. Leithold, Norbert 1073. Lenz, Siegfried 1073. Leupold, Dagmar 1073. Liersch, Werner 1074. Lillin, Andreas A. 1074. Loest, Erich 1074. Lorenc, Kito 1074. Maier, Andreas 1074. Manger, Jürgen von 1074. Mann, Frido 1074. Mann, Golo 1074. Marchwitza, Hans 1074. Margul-Sperber, Alfred 1074. Maron, Monika 1075. Marwig, Detlef 1075. Mayer, Hans 1075. Mayröcker, Friederike 1075. Meier, Gerhard 1075. Meinecke, Thomas 1075. Meinhold, Gottfried 1075. Meister, Ernst 1075. Menasse, Robert 1076. Mensching, Steffen 1076. Miehe, Ulf 1076. Mitgutsch, Waltraud Anna 1076. Modick, Klaus 1076. Mon, Franz 1076. Moníková, Libuše 1076. Mosebach, Martin 1077. Müller, Heiner 1077. Müller, Herta 1077. Mueller-Stahl, Armin 1078. Nadolny, Sten 1078. Neumeister, Andreas 1078. Nick, Dagmar 1079. Nöstlinger, Christine 1079. Nossack, Hans Erich 1079. Özdamar, Emine Sevgi 1079. Oliver, José Francisco A. 1079. Overath, Angelika 1079. Plenzdorf, Ulrich 1080. Pleschinski, Hans 1080. Pludra, Benno 1080. Politycki, Matthias 1080. Pollack, Martin 1080. Polt, Gerhard 1080. Priessnitz, Reinhard 1080. Puchner, Willy 1081. Puganigg, Ingrid 1081. Rakusa, Ilma 1081. Ransmayr, Christoph 1081. Rathenow, Lutz 1082. Rauner, Liselotte 1082. Reding, Josef 1082. Reinig, Christa 1082. Riccabona, Max 1082. Richter, Hans Werner 1082. Richter, Jutta 1082. Rinser, Luise 1083. Rosendorfer, Herbert 1083. Rühmkorf, Peter 1083. Runge, Erika 1083. Rychner, Max 1083. Schädlich, Hans Joachim 1083. Schallück, Paul 1084. Schernikau, Ronald M. 1084. Schimanek, Jürgen 1084. Schirmbeck, Heinrich 1084. Schirmer, Bernd 1084. 
Schlesinger, Klaus 1084. Schlink, Bernhard 1085. Schmidt, Arno 1085. Schmidt, Kathrin 1087. Schneider, Helga 1087. Schnur, Martin 1087. Schorlemmer, Friedrich 1087. Schreyer, Wolfgang $1087 . \quad$ Schrott, Raoul 1087. Schwarze, Hans Dieter 1088. Sebald, Winfried Georg 1088. Semmer, Gerd 1091. Simmel, Johannes Mario 1091. Sperr, Martin 1091. Steiner, Wilfried 1091. Sterchi, Beat 1091. Strauß, Botho 1091. Süskind, Patrick 1092. Şurdum, Kundeyt 1092. Tabori, George 1092. Thelen, Albert Vigoleis 1092. Thenior, Ralf 1094. Timm, Uwe 1094. Tumler, Franz 1094. Ujvary, Liesl 1094. Ulbrich, Bernd 1095. Valentin, Thomas 1095. Vollmer, Walter 1095. Waberer, Keto von 1095. Wackwitz, Stephan 1095. Wagner, Richard <* 1952〉 1095. Walser, Martin 1097. Walter, Otto F. 1097. Wander, Maxie 1097. Waterhouse, Peter 1097. Weil, Grete 1097. Weiss, Peter 1098. Weissglas, Immanuel 1098. Wenders, Wim 1098. Werner, Markus 1098. Weyrauch, Wolfgang 1098. Widmer, Urs 1098. Wiersch, Jürgen 1098. Wildenhain, Michael 1098. Wittkamp, Frantz 1098. Wodin, Natascha 1099. Wohmann, Gabriele 1099. Wolf, Christa 1099. Wolf, KlausPeter 1099. Wolf, Konrad 1099. Wolf, Ror 1099. Wollschläger, Hans 1099. Wühr, Paul 1100. Zahl, Peter-Paul 1100. Zwerenz, Gerhard 1100

\section{Gegenwartsliteratur}

Allgemeines 1100. Einzelne Abschnitte 1100. Lyrik 1101. Epik 1101. Drama 1103. Ackrill, Ursula 1103. Akın, Fatih 1103. Albrecht, Jörg 1103. Altenburg, Matthias 1103. Amir, Ibrahim 1103. Ani, Friedrich 1104. Arjouni, Jakob 1104. Arslan, Thomas 1104. Bach, Tamara 1104. Bärfuss, Lukas 1104. Banciu, Carmen-Francesca 1105. Bánk, Zsuzsa 1106. Bauer, Jutta 1106. Bauerdick, Rolf 1106. Becker, Artur 1106. Becker, Thorsten 1106. Berg, Sibylle 1106. Bergmann, Michel $1106 . \quad B i c k e r$, Björn 1106. Bieri, Peter 1107. Bilkau, Kristine 1107. Biller, Maxim 1107. Bleutge, Nico 1107. Bodrožić, Marica 1107. Borries, Friedrich von 1107. Bossong, Nora 1107. Brandt, Jan 1107. Brauns, Dirk 1107. Breznik, Melitta 1107. Bronsky, Alina 1107. Bruhns, Wibke 1108. Brussig, Thomas 1108. Büscher, Wolfgang 1108. Bullerdiek, Bolko 1108. Cotten, Ann 1108. Daher, Lydia 1108. Danz, Daniela 1108. Dinev, Dimitré 1108. Dorn, Thea 1108. Draesner, Ulrike 1108. Droste, Wiglaf 1109. Dückers, Tanja 1109. Düffel, John von 1109. Duve, Karen 1109. Ebmeyer, Michael 1109. Eckenga, Fritz 1109. Eckert, Guido 1109. Egger, Oswald 1109. Elmiger, Dorothee 1109. Engin, Osman 1109. Erpenbeck, Jenny 1109. Faktor, Jan 1111. Ferentschik, Klaus 1111. Franck, Julia 1111. Funke, Cornelia 1111. Fusek, Katja 1111. Gaertner, Joachim 1111. Gamillscheg, Marie 1111. Geiger, Arno 1111. Gerlof, Kathrin 1112. Gier, Kerstin 1112. Glattauer, Daniel 1112. Glavinic, Thomas 1112. Gorelik, Lena 1113. Grether, Kerstin 1114. Grimsen, Germar 1114. Grjasnowa, Olga 1114. Gruber, Sabine 1114. Grünbein, Durs 1114. Hacker, Katharina 1114. Haderlap, Maja 1114. Hadžibeganović, Alma 1114. Händler, Ernst-Wilhelm 1114. Hein, Jakob 1114. Heinemann, Elke 1114. Heinrich, FinnOle 1115. Hennig von Lange, Alexa 1115. Hermann, Judith 1115. Herrndorf, Wolfgang 1115. Hettche, Thomas 1115. Höhtker, Christoph 1115. Hollatko, Lizzy 1115. Hoppe, Felicitas 1116. Horváth, Martin 1116. Hotschnig, Alois 1116. Hulpe, Marius 1116. Hummel, Eleonora 1116. Ingendaay, Paul 1117. Janesch, Sabrina 1117. Jirgl, Reinhard 1117. Jonigk, Thomas 1117. Kálnay, Juliana 1117. Kames, Maren 1117. Kehlmann, Daniel 1117. Kermani, Navid 1118. Khider, Abbas 1118. Kim, Anna 1118. Klare, Jörn 1118. Klein, Georg 1118. Kluck, Oliver 1118. Klüger, Ruth 1119. Köhler, Barbara 1119. Köhler, Harriet 1119. Konecny, Jaromir 1119. Kracht, Christian 1119. Kratochwil, Germán 1120. Krausser, 
Helmut 1120. Krieger, Hans 1121. Kröger, Merle 1121. Kuhligk, Björn 1121. Kummer, Tom 1121. Kutscher, Volker 1121. Lebert, Benja$\min$ 1121. Lehr, Thomas 1121. Lendle, Jo 1121. Lewitscharoff, Sibylle 1122. Mahler, Nicolas 1122. Mansmann, Nora 1122. Margwelaschwili, Giwi 1122. Martenstein, Harald 1122. Martynova, Olga 1122. Maurer, Jörg 1122. Mayer, Gina 1122. Meier, Angelika 1122. Melle, Thomas 1122. Menasse, Eva 1123. Meyer, Clemens 1123. Miller, Phoebe Ann 1123. Möller, Steffen 1123. Moers, Walter 1123. Mora, Terézia 1123. Moradpour, Mehdi 1123. Müller, Tina 1123. Muszer, Dariusz 1123. Nadj Abonji, Melinda 1124. Naoura, Salah 1124. Nendza, Jürgen 1124. Niemann, Norbert 1124. Orzessek, Arno 1124. Ostermaier, Albert 1124. Otremba, Hendrik 1124. Peer, Alexander 1124. Peltzer, Ulrich 1124. Peters, Christoph 1125. Petrowskaja, Katja 1125. Petzold, Christian 1126. Peuckert, Tom 1126. Pollesch, René 1126. Popp, Steffen 1126. Poschmann, Marion 1126. Poznanski, Ursula 1126. Rabinovici, Doron 1126. Rabinowich, Julya 1126. Rajčić, Dragica 1126. Rammstedt, Tilman 1126. Rau, Milo 1127. Regener, Sven 1127. Reifenberg, Frank M. 1127. Reinhardt, Dirk 1127. Richter, Falk 1128. Rinck, Monika 1128. Ritzel, Ulrich 1128. Roche, Charlotte 1128. Rock, Zé do 1128. Röggla, Kathrin 1128. Ruge, Eugen 1129. Sander, Gregor 1129. Sargnagel, Stefanie 1129. Schätzing, Frank 1129. Schalansky, Judith 1129. Schimmelpfennig, Roland 1129. Schirach, Ferdinand von 1129. Schlingensief, Christoph 1129. Schmid, Eva-Maria 1130. Schneider, Robert 〈* 1961〉 1130. Scho, Sabine 1130. Schorlau, Wolfgang 1130. Schröder, Martin Z. 1130. Schulze, Ingo 1130. Schwab, Werner 1130. Seethaler, Robert 1130. Seiler, Lutz 1130. Senkel, Günter 1131. Setz, Clemens J. 1131. Singer, Lea 1131. Stamm, Peter 1131. Stanišić, Saša 1133. Stavarič, Michael 1133. Stein, Benjamin 1135. Steinaecker, Thomas von 1135. Steinhöfel, Andreas 1135. Sträter, Torsten 1135. Streeruwitz, Marlene 1135. Stuckrad-Barre, Benjamin von 1135. Svolikova, Miroslava 1135. Tawada, Yoko 1136. Tellkamp, Uwe 1136. Tessnow, Gregor 1136. Thome, Stephan 1136. Trojanow, Ilija 1136. Trompeter, Julia 1136. Urban, Simon 1136. Vanderbeke, Birgit 1136. Veiel, Andres 1136. Vertlib, Vladimir 1136. Veteranyi, Aglaja 1137. Wagner, David 1137. Wagner, Jan 1137. Walser, Alissa 1137. Wawerzinek, Peter 1137. Weber, Anne 1137. Weiler, Jan 1137. Welt, Wolfgang 1137. Wenzel, Christoph 1137. Werner, Florian 1137. Wiebicke, Jürgen 1138. Winkler, Ron 1138. Witzel, Frank 1138. Zaeri-Esfahani, Mehrnousch 1138. Zaimoğlu, Feridun 1138. Zaptçıoğlu, Dilek 1138. Zeh, Juli 1138. Zeiner, Monika 1139. Zelter, Joachim 1139. Zipfel, Daniel 1139. Zippert, Hans 1139

XXXVI. Theater-, Film-, Medienwissenschaft

XXXVII. Bibliothekswesen - Buchwesen - Verlagswesen 